\title{
Rolling Element Bearing Fault Diagnosis Using Integrated Nonlocal Means Denoising with Modified Morphology Filter Operators
}

\author{
Mien Van, Pasquale Franciosa, and Dariusz Ceglarek \\ Manufacturing Systems and Digital Lifecycle Management Group, WMG, University of Warwick, Coventry, UK \\ Correspondence should be addressed to Mien Van; vanmien1@gmail.com
}

Received 19 March 2016; Revised 12 May 2016; Accepted 4 October 2016

Academic Editor: Yaguo Lei

Copyright @ 2016 Mien Van et al. This is an open access article distributed under the Creative Commons Attribution License, which permits unrestricted use, distribution, and reproduction in any medium, provided the original work is properly cited.

\begin{abstract}
The impulses in vibration signals are used to identify faults in the bearings of rotating machinery. However, vibration signals are usually contaminated by noise that makes the process of extracting impulse characteristic of localized defect very challenging. In order to effectively diagnose bearing with noise masking vibration signal, a new methodology is proposed using integrated (i) nonlocal means- (NLM-) based denoising and (ii) improved morphological filter operators. NLM based denoising is first employed to eliminate or reduce the background noise with minimal signal distortion. This denoised signal is then analysed by a proposed modified morphological analysis (MMA). The MMA analysis introduces a new morphological operator which is based on Modified-Different (DIF) filter to include only fault relevant impulsive characteristics of the vibration signal. To improve further performance of the methodology the length of the structure element (SE) used in MMA is optimized using a particle swarm optimization- (PSO-) based kurtosis criterion. The results of simulated and real vibration signal show that the integrated NLM with MMA method as well as the MMA method alone yields superior performance in extracting impulsive characteristics of vibrations signals, especially for signal with high level of noise or presence of other sources masking the fault.
\end{abstract}

\section{Introduction}

Condition monitoring-diagnostic methods have an important role in increasing reliability and safety of mechanical systems [1-8]. Rolling element bearings are widely used components in rotating machines and their faults are one of the most frequent reasons for machine failures or performance deterioration (smooth and quiet running). Therefore, it is crucial to be able to quickly and accurately detect faults in rolling element bearings. Different measurements are used for detection and diagnosis of bearings faults such as acoustics, temperature, wear debris analysis, and vibration; though, the last one is the most widely used. By analysing the changes in vibration signals caused by faulty components, the root of faults can be identified [9-11].

In a mechanical system, when a rolling element strikes a localized defect, an impulse of short duration is generated. The impulses are generated periodically and their characteristics depend on the location of the localized defect in the bearing, that is, the outer race, the inner race, or the rolling element. Hence, the types of bearing fault can be effectively identified by extracting the fault related impulses in vibration signal. To identify the bearing characteristic frequencies (BCF) associated with faults in the bearing elements, envelop analysis has been developed [12]. In this method, first, the vibration signal is filtered around the mechanical resonance of the machine. Then, an amplitude demodulation technique and Fourier transform are applied to extract fault features. However, the selection of the filtering frequency band is a challenge in this approach. To effectively select the filtering frequency band, spectral kurtosis (SK) and its advanced methods $[13,14]$ have been developed.

In order to analyse the vibration signal, time-frequency analysis such as wavelet transform has been introduced as an efficient method [15-17]. However, using the wavelet transform, the performances are strongly dependent on the choice of the wavelet basis function. Only signal features that correlate well with the shape of the wavelet function have the 
potential to lead to high value coefficient. All other features may be completely ignored. Another time-frequency method widely used in vibration signal analysis is Hilbert-Huang Transform (HHT) [18-20]. This technique works by performing a timed adaptive decomposition operation, known as empirical mode decomposition (EMD), to process the signal. The signal is then decomposed into a set of complete and almost orthogonal components known as intrinsic mode functions (IMFs), which are almost a monocomponent. However, performing the EMD method is computationally expensive.

In recent years, a new nonlinear signal processing technique based on mathematical morphology (MM) has been developed. The MM is employed to decompose a signal into several physical parts according to the geometric characteristics of a certain structuring element (SE). The SE is a shape, used to probe with a given signal to evaluate how this shape fits or misses the shapes in the signal. The transformations of MM require only addition and comparison operators. Hence, they impose low computation burden, which is advantageous for real-time applications. The MM has been successfully applied for various aspects of signal processing [2123], including extracting impulsive component, for rolling element bearing fault diagnosis. Nikolaou and Antoniadis [24] introduced MM into fault diagnosis of rolling element bearing with a flat structure element (SE). They suggested that the length of SE should be 0.6 times the pulse repetition period for efficient extraction of fault frequency. Zhang et al. [25] proposed a multiscale to extract morphological features at different scales for bearing fault diagnosis. Wang et al. [26] adopted an improved morphological filter for vibration signal to extract the impulsive attenuation signals. In this approach, the SE is constructed in the form of impulsive attention, and a new criterion is suggested to optimize the SE. Li et al. [27] suggested a weighted multiscale morphological gradient filter for rolling element bearing fault detection to depress the noise at large scale and preserve the impulsive shape details at small scale. Work conducted by Dong et al. [28] indicated that the length of SE should be chosen based on the signal-to-noise ratio (SNR) criterion for efficient extraction of impulsive component. Raj and Murali [29] proposed a new algorithm for the SE selection based on kurtosis. In summary, several morphology-based analyses have been developed for roller bearing fault diagnosis; though most of them were designed based on Average (AVR) (5) or Different (DIF) (6) filters.

However, the AVR or DIF filters have been shown to be inadequate for extracting impulsive features in bearing fault diagnosis. In addition, the presence of high measurement noise results in morphology analyses, based on AVR and DIF, to capture a lot of noise impulses that are unrelated to the bearing faults. Therefore, the design of morphology analysis is difficult when it comes to extracting fault related impulses from such noise signal. In such cases, existing morphology analyses are inadequate. If the noise impulses are filtered out, then the morphology analysis becomes much more effective in extracting the faulty impulses from the denoised signal.

Denoising approach based on nonlocal means (NLM) introduced by Buades et al. in [30] is a data-driven diffusion mechanism. It has been widely and successfully applied for image denoising [31, 32]. The basic idea of the NLM algorithm is to measure the similarity between two pixels by evaluating the distance between small patches centered on these two pixels. In this way, the NLM method can provide denoising while minimizing signal distortion compared to the discrete wavelet transform [33]. The performance of NLM denoising for vibration signals has been studied and verified in our previous work [18]. In [18], the vibration signal is first denoised by using NLM. The denoised signal is then decomposed by EMD to extract the impulse characteristics. This approach, however, requires intensive EMD computation and was shown to be less effective for signals with high level noise.

In this paper, in order to effectively diagnose fault bearing with noise masking vibration signal, a new methodology is proposed using integrated (i) nonlocal means- (NLM-) based denoising of the vibration signal to eliminate or reduce unnecessary noise and (ii) modified morphological analysis (MMA) based on a novel filter operator (Modified-DIF filter) and real-time optimization of SE.

The purpose of the NLM denoising is to reduce or eliminate the impulse characteristics due to measurement noise [18]. The purified signal obtained through the NLM denoising is then analysed by MMA to extract the bearing fault related impulse characteristics.

As a next step the modified morphological analysis (MMA) is developed and used in order to effectively extract the impulse characteristics of the bearing fault in vibration signal with all other nonrelevant to bearing fault characteristics being suppressed to zero. This is achieved by defining a new morphological operator, called Modified-DIF filter, which has capability to include only impulse characteristics of the vibration signal with all other signal components being suppressed to zero.

Simultaneously, the lengths of SEs are automatically adjusted using particle swarm optimization (PSO) based kurtosis maximization rule. The PSO algorithm [34] is known as an efficient method in finding an optimal solution for the real optimization problems, such as optimizing the selected feature inputs [35] and optimizing the parameters of support vector machine (SVM) classifier [36]. By optimizing the length of SE, the proposed morphological operator (Modified-DIF filter) can maximize the number of extracted impulses and therefore has a better fault related impulse extracting capability compared with the existing AVR or DIF filter approaches.

The proposed method is tested through the simulated impulse attenuation signals and real bearing signal with different level of noises.

The results of simulated and real rolling element bearing vibration signal analyses show that the integrated NLM and MMA method as well as the MMA method alone yields superior performance in extracting impulsive characteristics of vibrations signals, especially for vibration signal with high level of noise or presence of other sources masking the vibration signal.

The rest of this paper is organized as follows. Section 2 introduces a new morphological operator and the criterion for SE selection based on PSO-based kurtosis criteria. The hybrid NLM denoising and MMA for roller bearing fault 
diagnosis is presented in Section 3. Section 4 shows the performance of the proposed method compared with the existing methods in impulse extracting capability for a simulated experiment signal. Section 5 shows the application of the proposed method to detect the fault using real bearing vibration data. Finally, conclusions are given in Section 6.

\section{Modified Morphology Analysis}

2.1. Theory Fundamental of Mathematical Morphology. The theory of the MM was first introduced as an image processing methodology by Serra [37]. Based on this idea, morphological filter with functional structure for one-dimensional time series data is then presented by Maragos and Schafer [38]. In morphological signal processing, the shape of the signal is modified by transforming it through its intersection with the structure element (SE).

Let $f(n)$ be the one-dimensional signal which is a discrete function over a domain $F=(0,1,2, \ldots, N-1)$. And let $g(m)$ be the SE, which is the discrete function over a domain $G=$ $(0,1,2, \ldots, M-1)$. The primary morphological operators are dilation and erosion.

Dilation is the operator that combines two sets using vector addition of set element and is defined as

$$
\begin{aligned}
(f \oplus g)(n)=\min [f(n-m)-g(m)], & \\
& \quad m \in 0,1,2, \ldots, M-1,
\end{aligned}
$$

where $\oplus$ denotes the operator of dilation.

Erosion is the morphological dual to dilation. It combines two sets using the vector subtraction of set elements and is defined as

$$
\begin{aligned}
(f \Theta g)(n)=\min [f(n+m)-g(m)], & \\
& m \in 0,1,2, \ldots, M-1,
\end{aligned}
$$

where $\Theta$ denotes the operator of erosion.

Based on the erosion and dilation operators, two other basic morphological operators, the opening and closing, can be further defined as

$$
\begin{aligned}
& (f \circ g)(n)=(f \Theta g \oplus g)(n) \\
& (f \circ g)(n)=(f \oplus g \Theta g)(n),
\end{aligned}
$$

where $\circ$ stands for the opening operator and $\bullet$ for the closing operator. The effects of the four operators in processing for an example time series by the flat structure element are shown in Figure 1.

According to the results of the examples, we can see that the erosion operator can smooth negative impulses and inhibit positive impulses, while the dilation operator can smooth positive impulses and inhibit negative impulses. The opening preserves negative impulses but levels positive impulses, while the closing operator preserves positive impulses but fills up the valleys. Thus, closing and opening can be applied to detect positive and negative impulses, respectively.
2.2. Morphological Operators. Based on the theory of MM, there are two factors that need to be defined for morphological analysis: the morphological operator and the SE [28]. In practice, morphological operators are chosen based on the prior knowledge of positive or negative peaks of the signal. However, it is difficult to obtain the prior knowledge of the positive or negative peaks of the signal. In this case, opening or closing operators cannot be used for extracting the impulsive component. If this is the case, two kinds of morphological operators that combine the operation of four basic operators have been proposed and widely used for impulsive extraction [21-26]:

(1) Average (AVR) filter

$$
\operatorname{AVR}(f)=\frac{(f \cdot g+f \circ g)}{2}
$$

(2) Different (DIF) filter

$$
\operatorname{DIF}(f)=f \cdot g-f \circ g
$$

The AVR filter can be used to flatten the positive and negative impulsive features, corresponding to the smoothing filter. The DIF filter can be used to extract the positive and negative impulsive features simultaneously [21].

Although the AVR or DIF filter can effectively extract the impulsive component, its performance is decreased due to the existence of other components (not impulsive component). In this paper, a modification of the DIF filter is proposed, namely, Modified-DIF filter, for better extracting impulsive characteristics. It is designed as follows:

(3) Modified-DIF filter

$$
\begin{aligned}
\text { Modified-DIF }(f)= & \left(f \bullet g_{1}-f \circ g_{1}\right) \\
& -\left(\left(f \bullet g_{1}\right) \circ g_{2}-\left(f \circ g_{1}\right) \bullet g_{2}\right),
\end{aligned}
$$

where $g_{1}$ and $g_{2}$ are different structure elements. The morphological operator in (7) includes two parts: (1) DIF filter $(f \bullet g-f \circ g)$ that is used to extract the positive and negative impulse characteristics simultaneously and (2) NOTDIF filter $\left(\left(f \bullet g_{1}\right) \circ g_{2}-\left(f \circ g_{1}\right) \bullet g_{2}\right)$ that is used to level the positive and negative impulse characteristics simultaneously. Hence, by subtracting from the DIF filter to the NOTDIF filter, the result contains only the impulse component; all other components are suppressed to zero. By this way, the impulse components can be clearly identified.

\subsection{Particle Swarm Optimization-Based Kurtosis Criteria for SE Selection}

2.3.1. Kurtosis Criteria for SE Selection. In addition to the morphological operators, the selection of SE also affects the processing results [25]. Generally, only when the shape and scale of the signal are matched to those of SE, the signal can be reserved. The basic shapes of SEs include flat, line, triangle, semicircle, polynomials, and cosine curves. According to Zhang et al. [23], the shapes of the SE have less effect on 


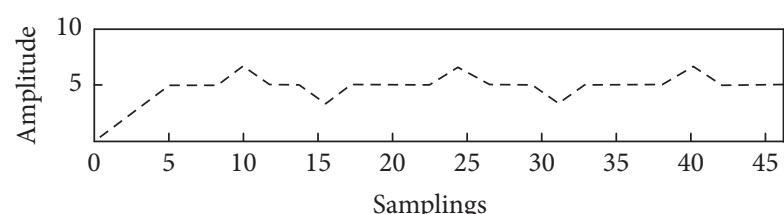

(a)

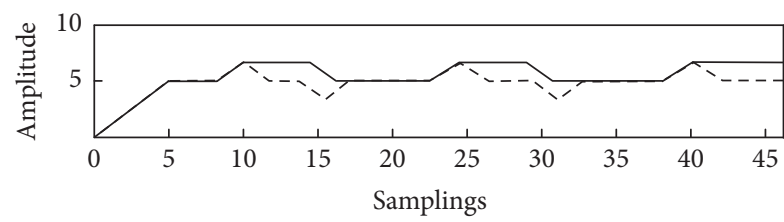

(c)

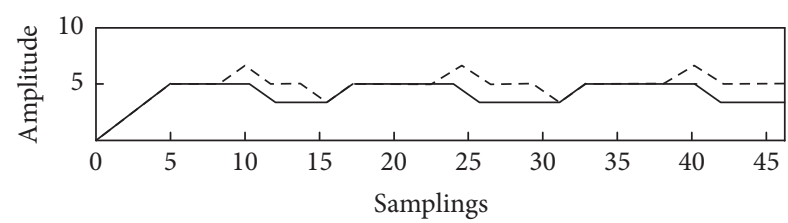

(b)

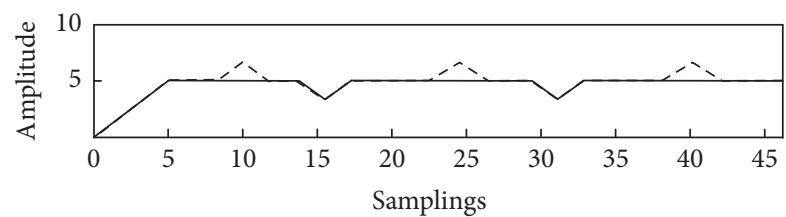

(d)

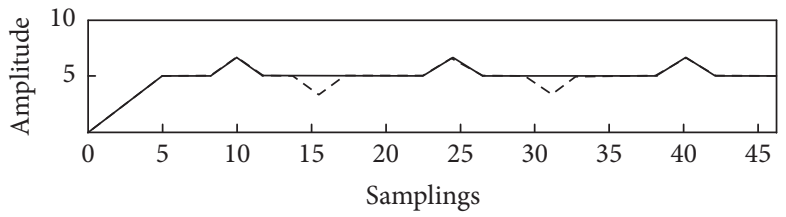

(e)

FIGURE 1: Signals produced from the applications of various morphological operators: (a) original signal; (b) signal after erosion; (c) signal after dilation; (d) signal after closing; and (e) signal after opening.

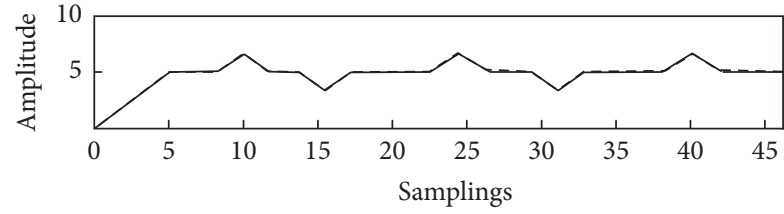

(a)

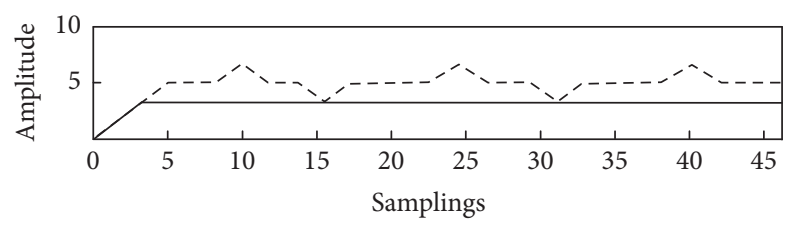

(c)

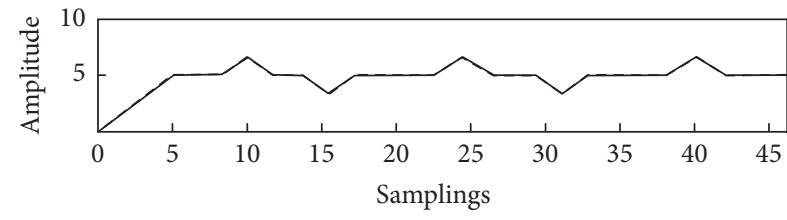

(b)

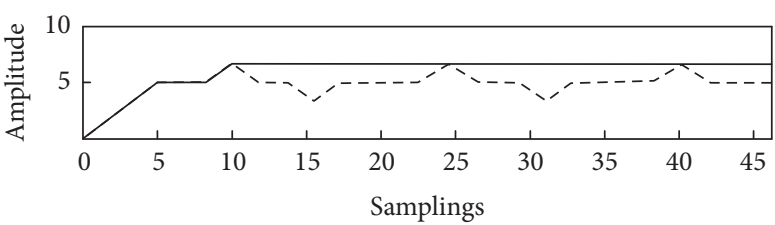

(d)

FIGURE 2: The results of using improper SEs lengths: (a) the filtered signal after using too short a length of SE for opening; (b) the filtered signal after using too short a length of SE for closing; (c) the filtered signal after using too long a length of SE for opening; (d) the filtered signal after using too long a length of SE for closing.

the analysis, so in order to simplify the computation, the flat $\mathrm{SE}$ is chosen in this study. And, in order to retain the shape characters of the signal entirely, all the height of the flat SE is defined as zero. The length of the SE is a crucial parameter for MM analysis. Considering much shorter length, much more impulsive features will be extracted from the signal while much noise will be retained, thereby making the demodulation of the signal difficult. On the other hand, with large length, few impulsive features will be extracted, and some useful information will be leveled. An illustration of effect of the improper SEs selection for MM analysis is shown in Figure 2.

The length around 0.6 times the pulse repetition period which proposed by Nikolaou and Antoniadis [24] is used in many studies. However, it does not suit all situations [28].
In this paper, kurtosis for SE's length selection is proposed. Kurtosis is used because of its sense to the number of impulses [25]. The larger value of the kurtosis indicates more number of peaks, which is an indication of the presence of the bearing fault. Hence the kurtosis criterion can be used to select the optimal length of SE. The definition of kurtosis is

$$
\operatorname{kurt}(y)=E\left(y^{4}\right)-3\left[E\left(y^{2}\right)\right]^{2},
$$

where $y$ is the sampled time series and $E$ represents the mathematical expectation of the series.

2.3.2. Selecting the Lengths of SE Using PSO. Particle swarm optimization (PSO) is a well-known optimization method based on population-based computation technique [34]. 
Compared to other heuristic optimization techniques such as genetic algorithm (GA), PSO possess several advantages such as simple concept, easy implementation, robustness to control parameters, and computational efficiency.

In PSO, population is called swarm and individuals are called particles. The $i$ th particle is characterized by its current position vector $x_{i}(t)=\left(x_{i 1}(t), x_{i 2}(t), \ldots, x_{i D}(t)\right)$ in the search space, where $D$ is the dimensionality of the search space, and velocity vector $v_{i}(t)=\left(v_{i 1}(t), v_{i 2}(t), \ldots, v_{i D}(t)\right)$. Each particle maintains a record of its personal best position $x_{\text {best }, i}(t)=\left(x_{\text {best }, i 1}(t), x_{\text {best }, i 2}(t), \ldots, x_{\text {best }, i D}(t)\right)$, and the whole swarm of particles maintains a record of global best position $G_{\text {best }, i}(t)=\left(g_{\text {best }, i 1}(t), g_{\text {best }, i 2}(t), \ldots, g_{\text {best }, i D}(t)\right)$. Particles move in the search space to search for the optimal solution. During the movement, each particle updates its position and velocity according to the distance to its personal best position and the distance to the global best position by the following equations:

$$
\begin{aligned}
v_{i d}^{t+1}= & \omega * v_{i d}^{t}+c_{1} r_{1} *\left(p_{\text {best }, i d}^{t}-x_{i d}^{t}\right)+c_{2} * r_{2} \\
& *\left(g_{\text {best }, i d}^{t}-x_{i d}^{t}\right) \\
x_{i d}^{t+1}= & x_{i d}^{t}+v_{i d}^{t+1},
\end{aligned}
$$

where $t$ represents the $t$ th iteration in the evolutionary process. $d \in D$ represents the $d$ th dimension in the search space. $\omega$ is the inertia weight. $c_{1}$ and $c_{2}$ are the acceleration constants. $r_{1}$ and $r_{2}$ are random values uniformly distributed in $[0,1]$.

In this paper, the optimal length of SE $g_{1}$ and $g_{2}$ are selected based on PSO. Four-real-code PSO is employed here to encode the length for each SE. For example, for one individual $\operatorname{SE}\left(g_{1}\right)=0.4356 T$ and $\operatorname{SE}\left(g_{2}\right)=0.7801 T$ ( $\operatorname{SE}\left(g_{i}\right) i=1,2$ is the length of SE $g_{i}$ and $T$ is the pulse repetition period), the length of SEs is encoded according to a position of PSO, as follows:

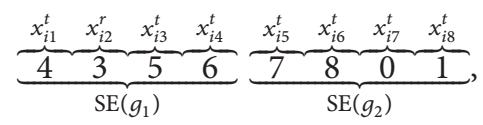

where $x_{i d}^{t} \in[0,9], d=1, \ldots, 8$ is the position of particle $i$ at iteration $t$ in the $d$ th dimension.

The procedure that describes the proposed SE's length selection is as follows.

Step 1. Initialize the parameters for PSO including swarm size, maximum iteration number, inertia weight, and velocity range.

Step 2. Initialize the positions $x_{i}$ and velocities $v_{i}$ of each particle.

Step 3. For each position $x_{i}$ of the particle from swarm, filter the signal by MMA associated with the lengths of SEs. Then, compute the corresponding fitness function. In this paper, fitness function is the kurtosis value of the filtered signal.

Step 4. Evaluate the fitness value of each initialized particle. Set the current position of each particle as its individual best position $p_{\text {best }, i}$. Set the particle position with the best fitness value in the swarm as the global best position $G_{\text {best }}$.

Step 5. Update the velocity and position of each particle with (9) and (10) to generate a new swarm.

Step 6. For each candidate particle $p_{i}$ of new swarm, filter the signal with MMA and compute the corresponding fitness function.

Step 7. Evaluate the fitness value of the newly updated particles, and then update $p_{\text {best }, i}$ and $G_{\text {best }}$ of the swarm. For an individual particle, if the newly updated fitness value is smaller than the history local best value, the local best position $p_{\text {best, } i}$ would be replaced by its current position. For the swarm of particles, if the current fitness value is smaller than the global best one, the global best position $G_{\text {best }}$ would be replaced by the current position.

Step 8. If the maximum number of iterations is not yet reached, return to Step 5 .

Step 9. Select the best global position $G_{\text {best }}$ in the swarm and filter signal with corresponding MMA. For example, if the best global position $G_{\text {best }}=[0,1,3,4,9,2,7,8]$, the optimal length of SE $g_{1}$ is $0.0134 T$ and $g_{2}$ is $0.9278 T$.

\section{Integrated Nonlocal Means Denoising and Modified Morphology Analysis}

3.1. Nonlocal Means (NLM) Algorithm. The NLM filter is based on the assumption that signal content is likely to repeat itself within some neighborhood and in neighboring frame [30]. Starting from a true signal, $u$, a noise observation of signal $y$ is defined as $y=u+n$, where $n$ is an additive noise. The NLM denoised values, $\widehat{u}(i)$, at the sample, $i$, are obtained by a weighted average of all samples in their neighborhood $\Omega_{i}$ :

$$
\widehat{u}(i)=\frac{1}{M(i)} \sum_{j \in \Omega_{i}} \omega(i, j) y(i),
$$

where $M(i)=\sum_{j \in \Omega_{i}} \omega(i, j)$ is a normalization constant. The weights, $\omega(i, j)$, meet the conditions $0<\omega(i, j)<1$ and $\sum_{j} \omega(i, j)=1$, which compare the neighborhood of samples $i$ and $j$. The weight, $\omega(i, j)$, takes a large value if the neighborhood of sample $i$ is similar to the neighborhood of sample $j$; otherwise it takes a small value. It is computed as $[18,36]$

$$
\omega(i, j)=\exp \left(-\frac{\sum_{\lambda \in \Delta}(y(i+\lambda)-y(j+\lambda))^{2}}{B_{\Delta} 2 \delta^{2}}\right) .
$$

Here, $\delta$ is a bandwidth parameter, $\Delta$ represents a local patch of samples surrounding $i$, and $B_{\Delta}$ is its total sample. $d^{2}=$ $\sum_{\lambda \in \Delta}(y(i+\lambda)-y(j+\lambda))^{2}$ denotes the sum of the squared pointto-point dissimilarity between the sample in the patches centered on $i$ and $j$. 


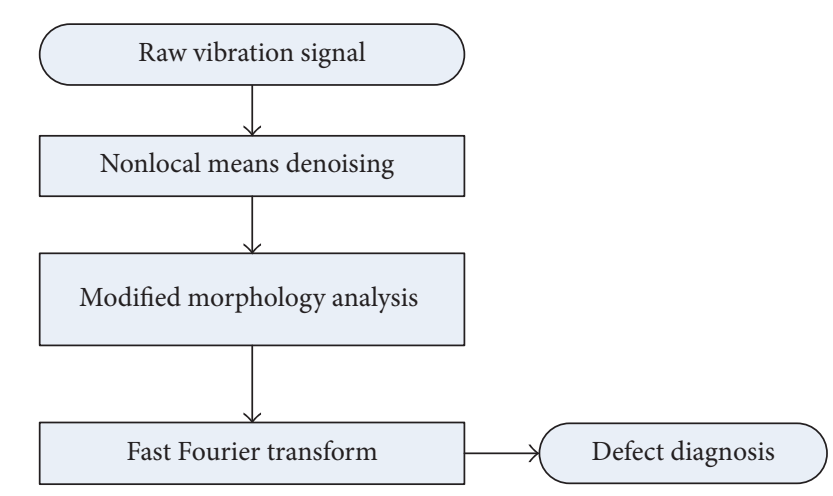

FIGURE 3: Flowchart of the integrated defect feature extraction technique.

3.2. NLM Parameters Selection. The performance of the NLM denoising is required to the selection of the NLM parameters. There are three NLM parameters: the patch size $B_{\Delta}$ (specified as a half-width $K, B_{\Delta}=2 K+1$ ), the size of neighborhood $\Omega_{i}$ (specified as a half-width $P, \Omega_{i}=2 P+1$ ), and the bandwidth $\delta$. These parameters can be effectively selected based on the analysis in our previous work [18].

\subsection{Hybrid NLM Denoising and MMA for Bearing Fault} Diagnosis. When a fault in one surface of a bearing strikes another surface, an impulsive of short duration is created. The characteristics of the impulses depended on the location of the localized defect, that is, the outer race, the inner race, or the rolling element. By correctly extracting such impulsive characteristics, the types of roller bearing fault can be effectively identified. Morphology analysis is an efficient tool for impulsive extraction. It has been widely applied to roller bearing fault diagnosis [23-29]. However, in the presence of noise impulses, the morphology analysis will extract both the fault related impulses and noise impulses. Consequently, the fault detection results based on the extracted impulses are sometime failing because the peaks corresponding to the noise impulses are sometime bigger than the peaks corresponding to the bearing characteristic impulses.

In order to enhance the performance of roller bearing fault diagnosis, a hybrid NLM denoising and MMA is proposed in this paper. The purpose of the NLM denoising is to eliminate or at least reduce the noise impulses due to the measuring device. The purified signal obtained through the NLM denoising is then analysed by MMA to correctly extract the fault related impulses. By this way, the fault diagnosis result for roller bearing fault diagnosis is more credible. The flowchart of the proposed fault diagnosis is shown in Figure 3.

\section{Simulated Experiments}

4.1. Simulated Signal Analysis. In order to illustrate the superior performance of the MMA as well as hybrid NLM denoising and MMA analysis, a simulated vibration signal is derived to analyse and compare with the existing methods such as morphological operators based on AVR or DIF. The simulated signal consists of three components [18]:

$$
x(t)=x_{1}(t)+x_{2}(t)+x_{3}(t),
$$

where $x_{1}(t)$ denotes a series of exponential decaying pulses (the periodic frequency is $11 \mathrm{~Hz}$ and the impulsive function in one period is $\left.5 e^{-10 t} \sin (10 \pi t)\right)$. Figure $4(\mathrm{a})$ shows the waveform of the $x_{1}(t) \cdot x_{2}(t)=\cos (2 \pi 15 t)+1.2 \cos (2 \pi 115 t)$ denotes the harmonic signal and is shown in Figure 4(b). Figure 4(c) shows the mixed signal of the impulsive signal and harmonic signal $\left(x_{1}(t)+x_{2}(t)\right) \cdot x_{3}(t)$ represents the generated Gaussian white noise. Considering the general situation, the low level (the standard deviation is 0.5 ) and the high level (the standard deviation is 3) Gaussian white noise signal are used here. The sampling frequency is $1024 \mathrm{~Hz}$, and the length is 1024 . In comparison with the real bearing vibration signal, $x_{1}(t)$ represents the impulsive signal which is generated by either the inner race or outer race or rolling elements; $x_{2}(t)$ and $x_{3}(t)$ represent the noise effects due to other machine elements and the measuring device, respectively.

Figure 5 shows the FFT spectrum of the composite signal in low level and high level Gaussian white noise. From this figure, the impulsive frequency $11 \mathrm{~Hz}$ and its harmonic frequencies, such as 22,33 , and $44 \mathrm{~Hz}$, are obtained. However, the harmonic frequencies 15 and $115 \mathrm{~Hz}$ are high at the same time.

In bearing fault diagnosis, the target is to extract the impulsive frequencies (i.e., $11 \mathrm{~Hz}$ and $22 \mathrm{~Hz}$ ), while reducing the harmonic frequencies (i.e., $15 \mathrm{~Hz}$ and $115 \mathrm{~Hz}$ ) and eliminating the white noise due to the measuring devices. In the following subsection we analyse the performance of the morphological operators based on AVR filter, DIF filter, proposed Modified-DIF filter, and proposed Modified-DIFbased NLM denoising filter in extracting impulsive features.

The morphology methods based on AVR filter, DIF filter, Modified-DIF filter, and Modified-DIF-based NLM denoising are used to analyse the simulated signals. Flat SEs are selected for the analysis, and the optimal length of the SEs is automatically determined by the maximum kurtosis criterion using PSO. The parameters of the NLM are selected as $K=20$ and $P=1000$. According to bandwidth selection procedure, the bandwidth value is selected as $\delta=0.6 \widehat{\sigma}$, 


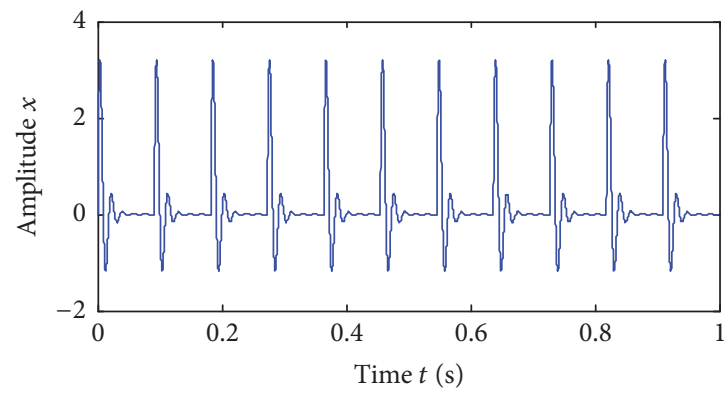

(a)

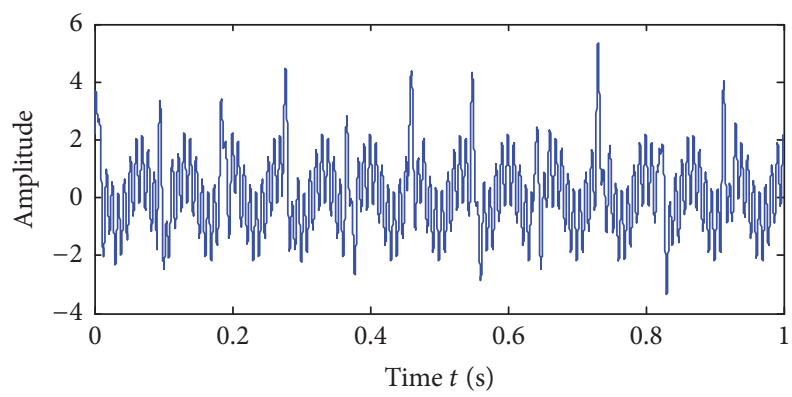

(c)

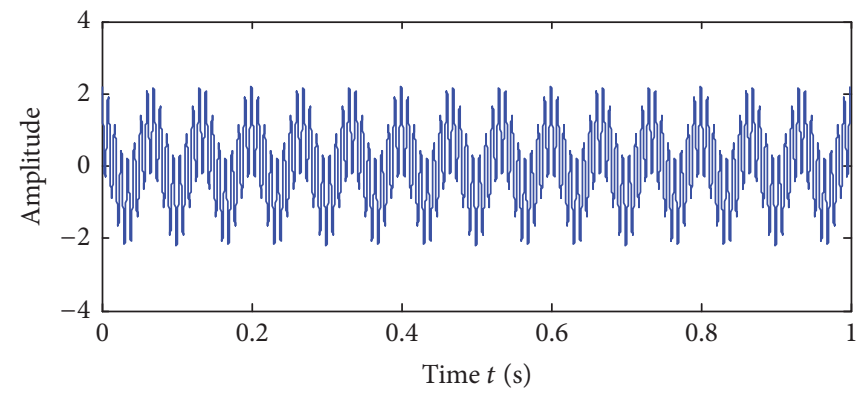

(b)

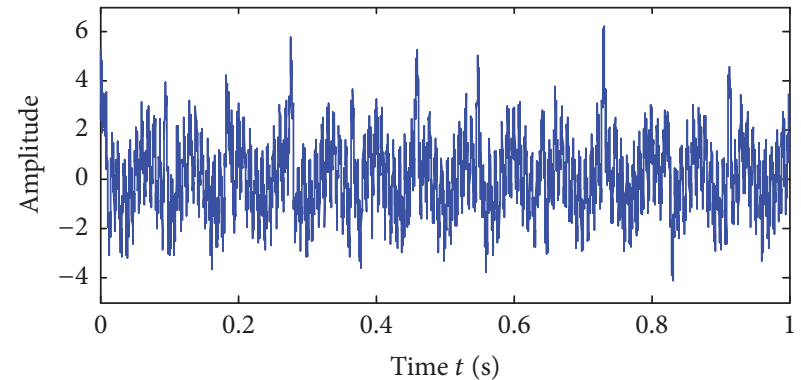

(d)

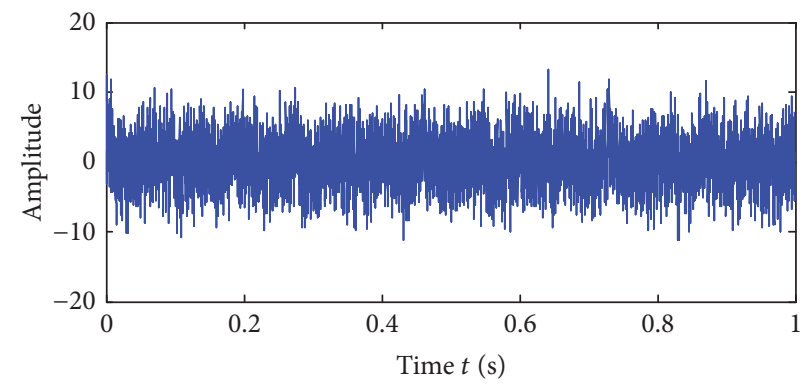

(e)

Figure 4: Waveform of simulated signal: (a) impulsive signal; (b) harmonic signal; (c) sum of impulsive signal and harmonic signal $\left(x_{1}(t)+\right.$ $\left.x_{2}(t)\right)$; (d) composite signal with low level white noise; (e) composite signal with high level white noise.

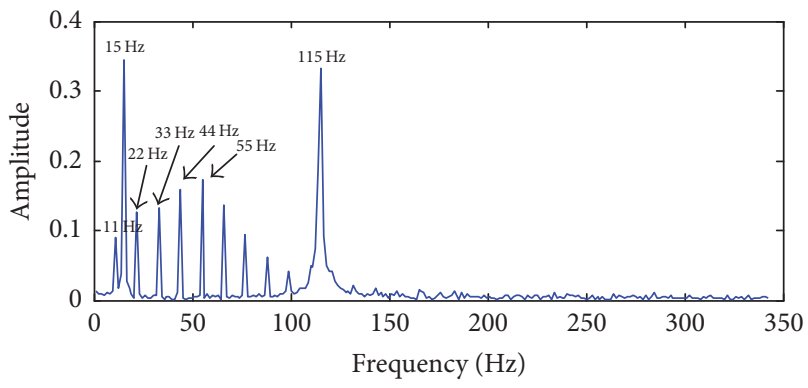

(a)

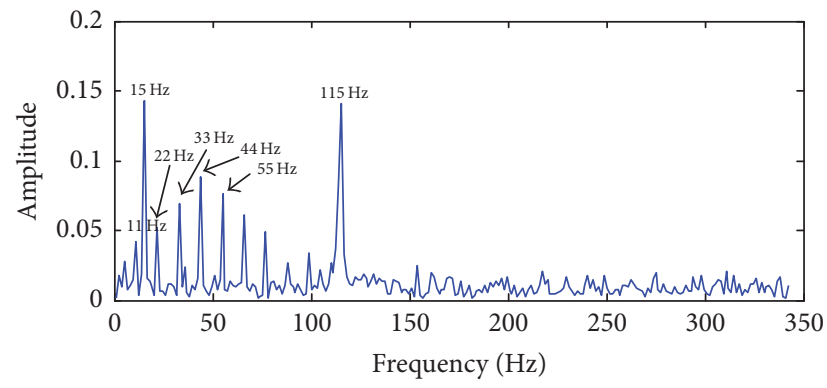

(b)

FIGURE 5: FFT spectrum of the simulated signal: (a) composite signal with low level Gaussian white noise and (b) composite signal with high level Gaussian white noise.

where $\widehat{\sigma}$ is an estimated noise standard deviation [18]. The comparison results among morphology analyses in the case of low level Gaussian white noise are shown in Figure 6, and in the case of high level Gaussian white noise, they are shown in Figure 7.
From Figure 6, the extracted impulsive component by Modified-DIF filter, shown in Figure 6(e), is better than that of DIF and AVR filters shown in Figures 6(a) and 6(c). As a result, the impulsive frequencies corresponding to the peaks are $11 \mathrm{~Hz}$ and its harmonics are $22,33 \mathrm{~Hz}$, and 


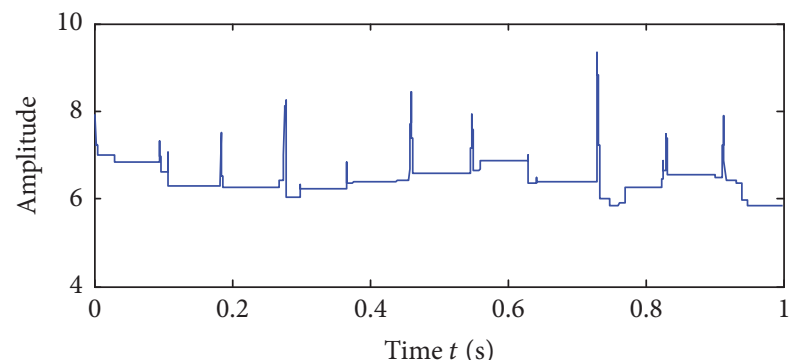

(a)

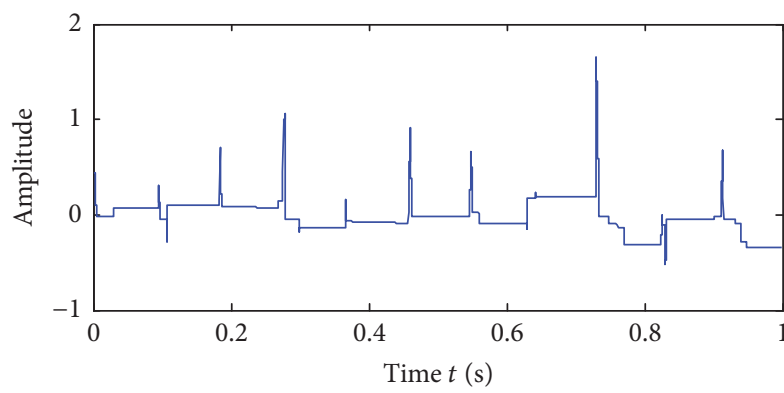

(c)

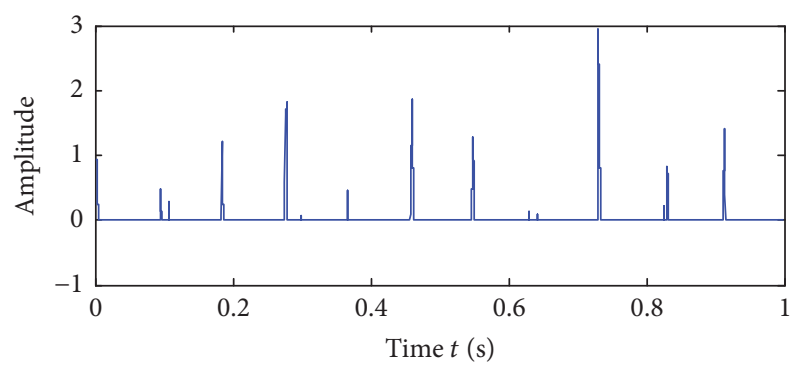

(e)

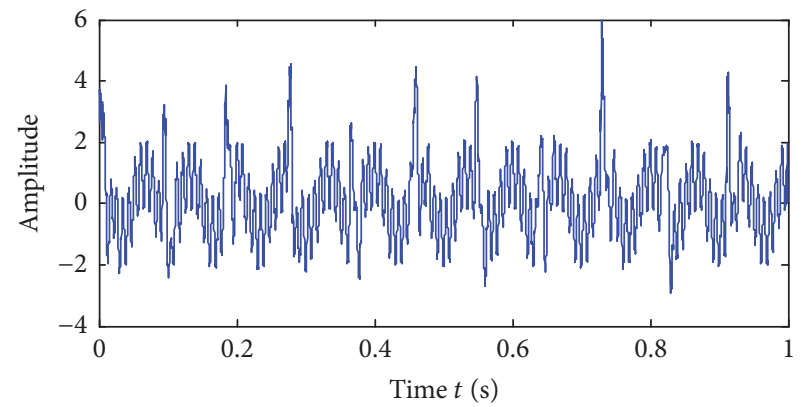

(g)

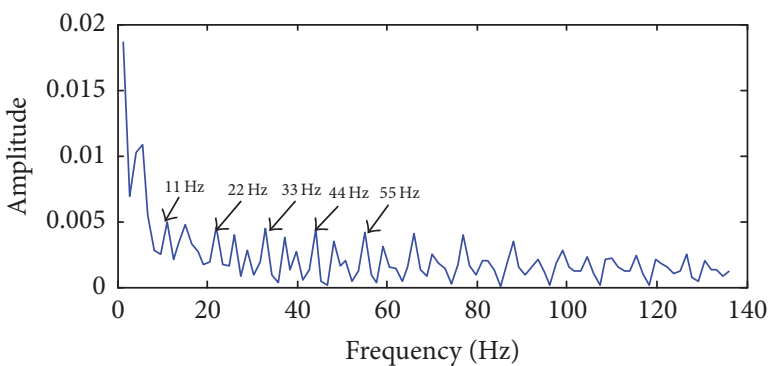

(b)

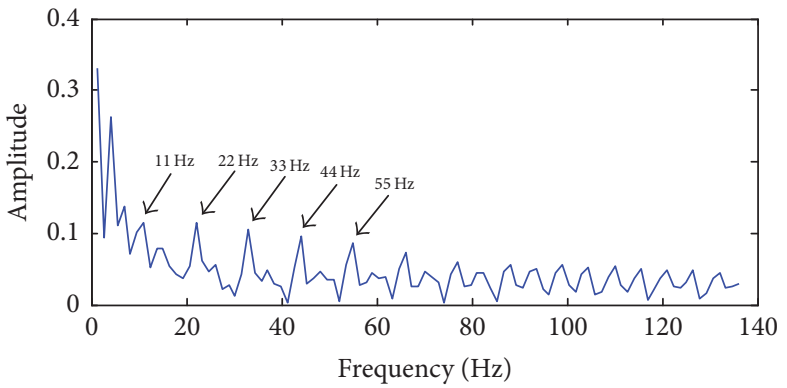

(d)

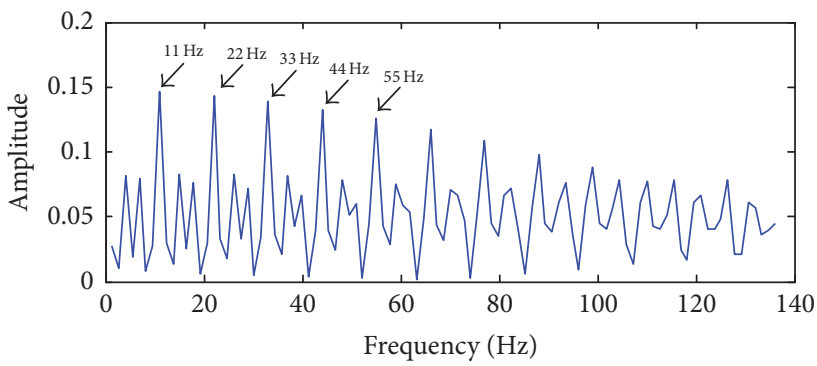

(f)

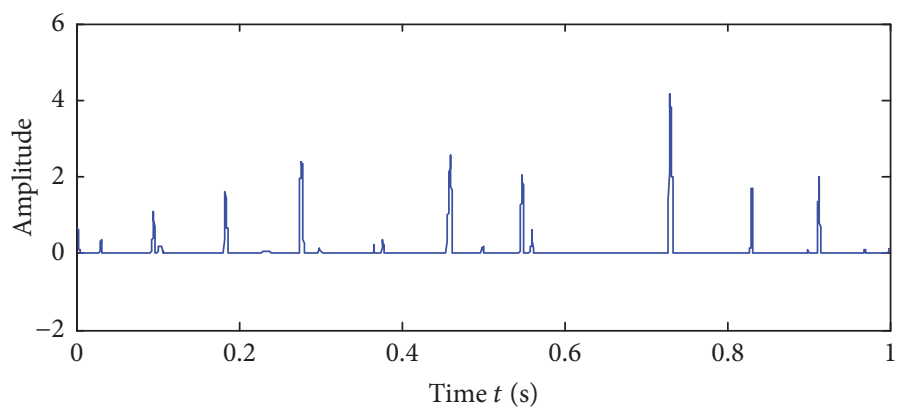

(h)

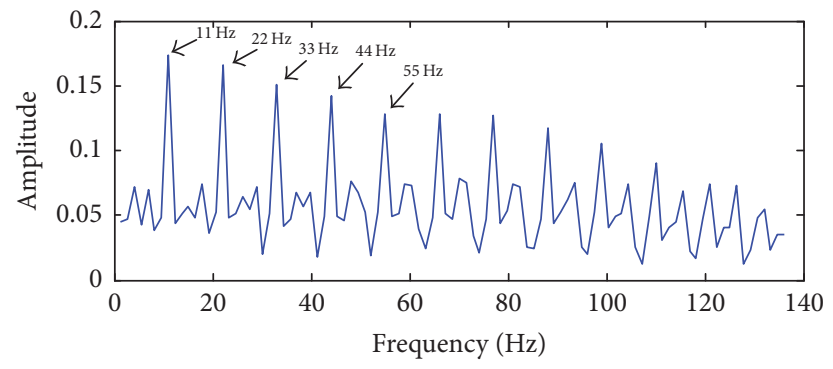

(i)

FIGURE 6: Simulated signal with low level Gaussian white noise: (a) waveform and (b) FFT spectrum of the simulated signal after DIF morphology analysis; (c) waveform and (d) FFT spectrum of the simulated signal after AVR morphology analysis; (e) waveform and (f) FFT spectrum of the simulated signal after Modified-DIF morphology analysis; (g) simulated signal after NLM denoising; (h) waveform; and (i) FFT spectrum of the simulated signal after Modified-DIF morphology-based NLM denoising analysis. 


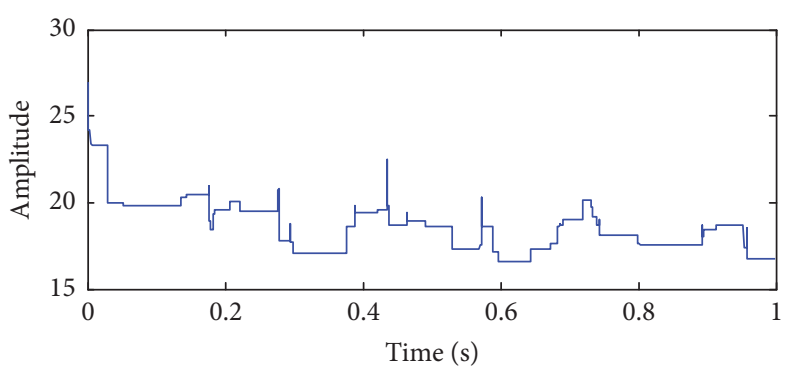

(a)

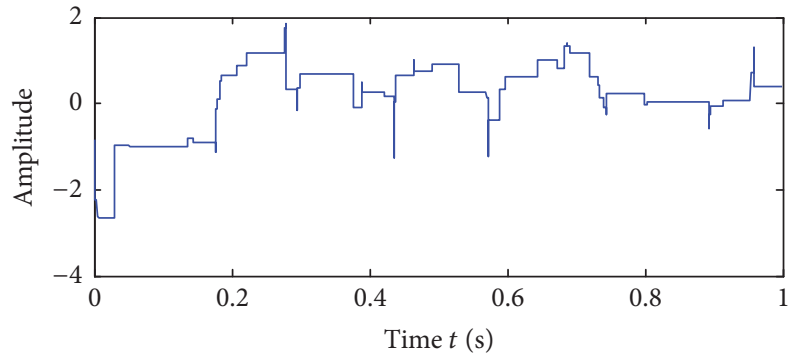

(c)

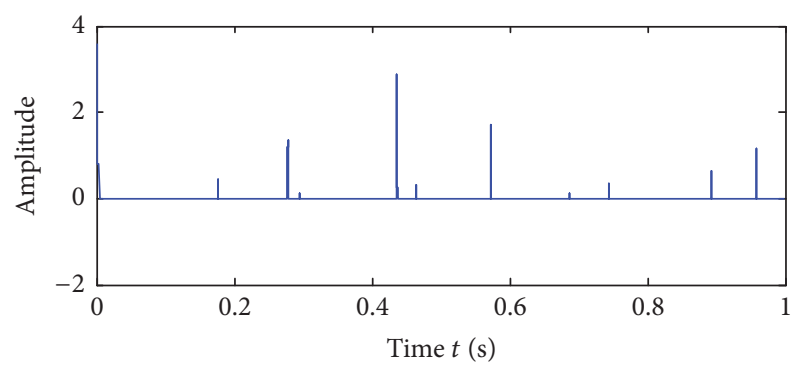

(e)

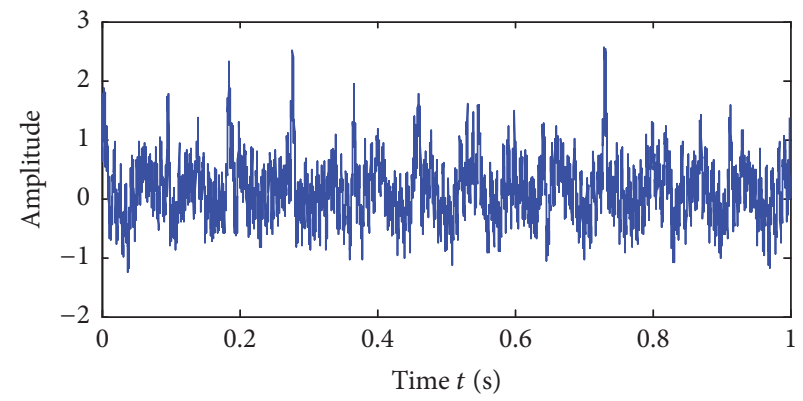

(g)

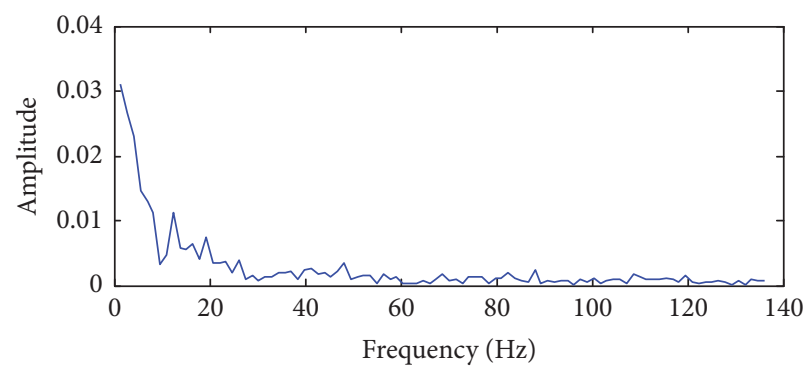

(b)

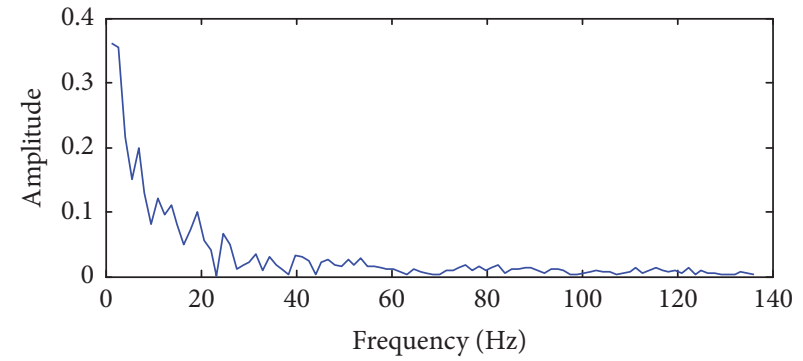

(d)

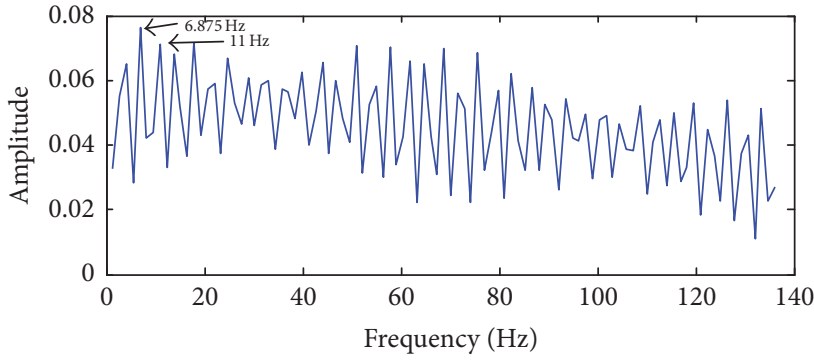

(f)

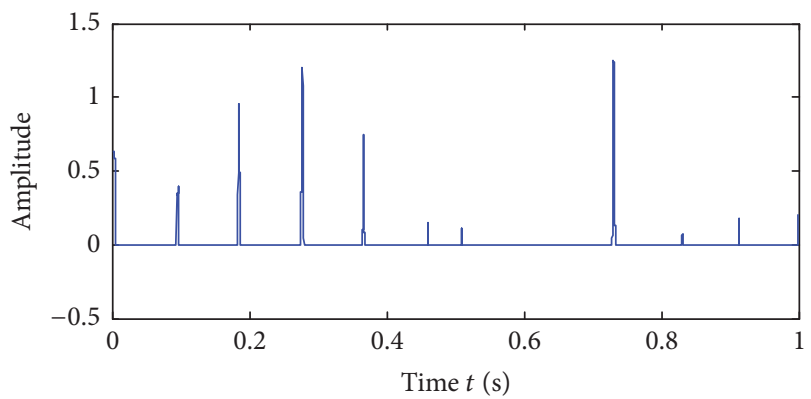

(h)

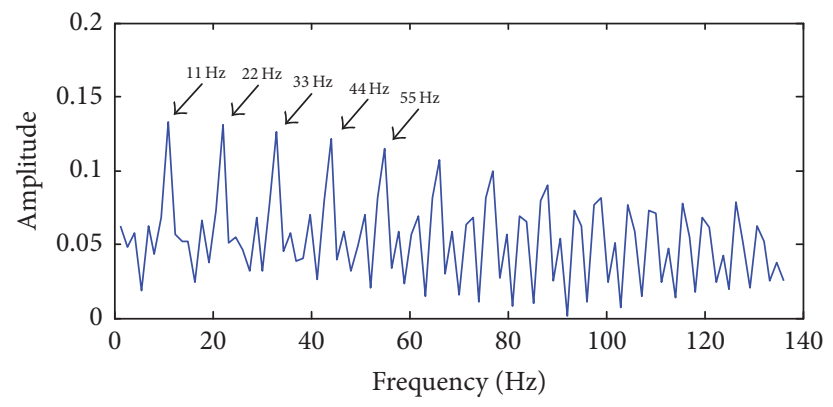

(i)

FIGURE 7: Simulated signal with high level Gaussian white noise: (a) waveform and (b) FFT spectrum of the simulated signal after DIF morphology analysis; (c) waveform and (d) FFT spectrum of the simulated signal after AVR morphology analysis; (e) waveform and (f) FFT spectrum of the simulated signal after Modified-DIF morphology analysis; (g) simulated signal after NLM denoising; (h) waveform; and (i) FFT spectrum of the simulated signal after Modified-DIF morphology-based NLM denoising analysis. 


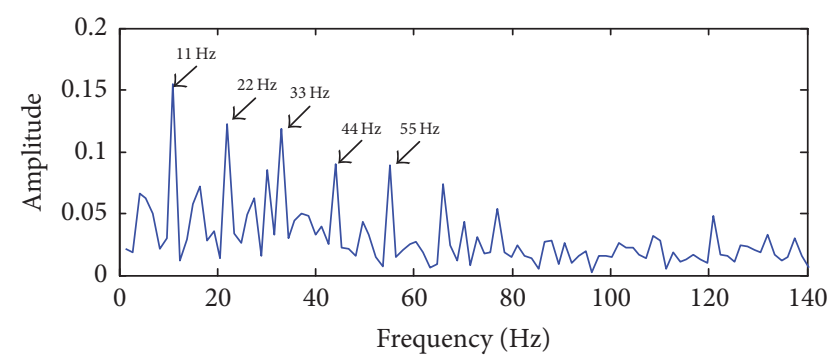

(a)

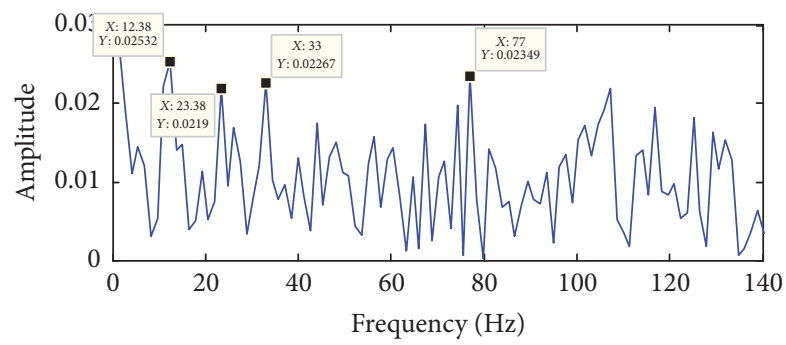

(b)

FIGURE 8: Simulated signal: (a) FFT spectrum of the simulated signal after NLM-EMD for low level Gaussian white noise and (b) FFT spectrum of the simulated signal after NLM-EMD for high level Gaussian white noise.

so on, reflected in the FFT spectrum of simulated signal. Using Modified-DIF morphology analysis (Figure 6(f)) is more obvious than the FFT spectrum of simulated signal using DIF or AVR morphology analysis (Figures 6(b) and 6(d)). By using NLM denoising to denoise the Gaussian white noise signal before applying the Modified-DIF morphology analysis (the denoised signal shown in Figure $6(\mathrm{~g})$ is very close to the original signal shown in Figure 4(c)), the FFT spectrum shown in Figure 6(i) is more obvious than the morphology analysis without denoising.

In the case of high level Gaussian white noise, the difference among morphology analyses is more obvious. In this case, the impulse component, which is extracted by using morphology analysis based on the AVR filter and DIF filter, is very weak, as shown in Figures 7(a) and 7(c). Consequently, the impulses reflected in their FFT spectrum are not identifiable, as shown in Figures 7(b) and 7(d). Using the morphology analysis based on the Modified-DIF operator, the impulse component is better extracted, as shown in Figure 7(e). However, due to the effect of the noise impulses, the peak $11 \mathrm{~Hz}$ and its harmonic are hidden by the peaks of noise frequencies, as shown in Figure 7(f). In this case, the application of the NLM denoising method is very necessary. The denoised signal after using NLM denoising is shown in Figure $7(\mathrm{~g})$ and the Gaussian white noise is much decreased. Using morphology analysis for this denoised signal, the faulty impulses are much better extracted, as shown in Figure 7(h). Therefore, the peak $11 \mathrm{~Hz}$ and its harmonics $22,33 \mathrm{~Hz}$, and so on can be found clearly in the FFT spectrum of the signal after hybrid NLM denoising analysis and MMA, as shown in Figure 7(i).

To further show the effectiveness of the hybrid NLM denoising analysis and MMA, we compare its performance with the approach NLM-EMD [18]. The FFT spectrums of the signal after the NLM-EMD for the low and high level noise signals are shown in Figures 8(a) and 8(b), respectively. From Figure 8 we can see that the NLM-EMD can extract the faulty impulses effectively for low level noise signal but fail for high level noise signal. In contrast, the proposed hybrid NLM denoising analysis and MMA is effective for both low and high level noise signal, as shown in Figures 6(i) and 7(i).

The results verify that the proposed hybrid NLM and MMA method is more effective in impulse extraction for localized fault bearing, especially in scenarios with high noise signals.

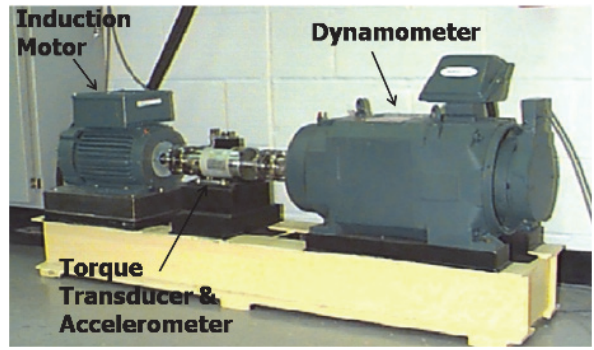

FIGURE 9: Experiment setup for vibration monitoring.

TABLE 1: Specification of bearing 6205-2RS-JEM SKF.

\begin{tabular}{lc}
\hline Parameters & Values (in.) \\
\hline Inside diameter & 0.9843 \\
Outside diameter & 2.0472 \\
Thickness & 0.5906 \\
Ball diameter & 0.3126 \\
Pitch diameter & 1.537 \\
Number of ball $(N)$ & 9 \\
Contact angle $(\alpha)$ & 0 \\
\hline
\end{tabular}

\section{Application on Defective Rolling Element Bearing}

In this section, the performance of the proposed methods based on MMA and hyrbrid NLM denoising and MMA is verified through the real roller bearing vibration signal. The bearing vibration data used in this experiment are taken from the benchmark data, Case Western Reserve University Bearing Data Center (2013) [39]. Figure 9 shows the setup of the experiment to collect the vibration signal. The system includes a $2 \mathrm{hp}$ motor (left), a torque transducer/encoder (center), a dynamometer (right), and control electronics (not shown). The test bearings are put in the output of the motor shaft, as shown in the figure. Table 1 displays the specification of the testing bearings in the experiment setup. The bearing faults are generated by using electrodischarge machining. The accelerometers, which are placed at the 3 oclock position on the drive end of the motor housing, are used to collect the vibration signal. The data are collected at the frequency 


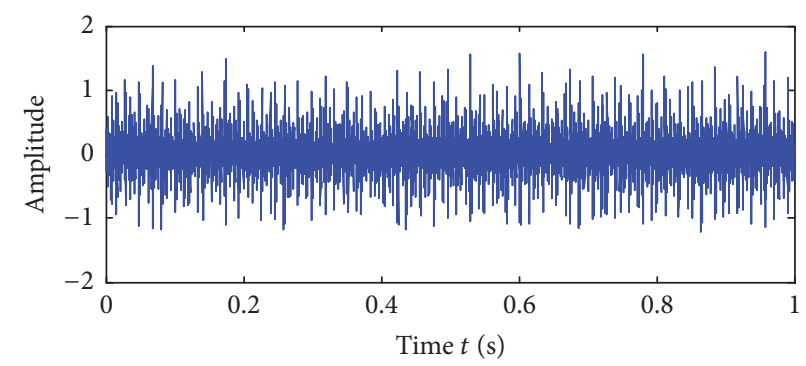

FIGURE 10: Waveform in the time domain of the vibration signal from inner race defect.

of $12,000 \mathrm{~Hz}$. The rotation frequency of the motor is $F_{r}=$ $29.95 \mathrm{~Hz}$.

Based on the principle operation of the bearing, when the motor shaft is rotating, the friction will be generated between inner race, ball, and outer race. Depending on the geometry of the bearing, the friction will create the impulse vibration signal at specific frequency. Hence, different specific frequencies will be generated according to the source of the fault, that is, inner race, outer race, or ball fault. The characteristic frequencies for IR fault $f_{i}$ and OR fault $f_{o}$ are formulated as

$$
\begin{aligned}
& f_{i}=\frac{N}{2} F_{r}\left(1+\frac{d}{D} \cos \alpha\right) \\
& f_{o}=\frac{N}{2} F_{r}\left(1-\frac{d}{D} \cos \alpha\right),
\end{aligned}
$$

where $N$ denotes the number of ball, $F_{r}$ denotes the rotation frequency, $d$ denotes the diameter of the ball, $D$ is the pitch diameter, and $\alpha$ is the contact angle.

5.1. Application on Inner Race Fault Detection. In this section, the performance of the proposed methods for detecting the inner race fault is shown. From (15), when the shaft rotational speed is $1797 \mathrm{rpm}$, the characteristic frequency for the inner race fault is obtained as $162.19 \mathrm{~Hz}$. The time domain of the recorded vibration signal is shown in Figure 10.

Figure 11 shows the performance of the MMA and hybrid NLM denoising and MMA. From Figure 11(a) which shows the waveform of the inner race vibration signal after MMA, the impulses generated by the inner race fault are easy to identify in the filtered signal compared to the original one shown in Figure 10. Figure 11(b) shows the FFT spectrum of the MMA filtered signal. It is obvious to see that the peaks are obtained at frequency $f_{i}=161.1 \mathrm{~Hz}$. The obtained peak value is very close to the calculated characteristic frequency, $162.19 \mathrm{~Hz}$. In addition, the harmonic frequency $2 f_{i}=323.7 \mathrm{~Hz}$ is visible clearly. Other peaks are identified to $2 F_{r} \approx 60.06 \mathrm{~Hz}, f_{i}-2 F_{r} \approx 102.5 \mathrm{~Hz}, f_{i}+2 F_{r} \approx$ $221.2 \mathrm{~Hz}$, and $2 f_{i}-2 F_{r} \approx 263.7 \mathrm{~Hz}$. However, due to the effects of noise, many noise impulses and the small peaks corresponding to noise impulses existed in the MMA filtered signal and FFT spectrum, respectively. To suppress these noise impulses, NLM denoising is applied. The denoised signal obtained by NLM denoising is shown in Figure 11(c).
Compared to the original signal shown in Figure 10, the noise component has been diminished. As a result, the FFT spectrum (shown in Figure 11(e)) of the signal after hybrid NLM denoising and MMA (shown in Figure 11(d)) contains few peaks corresponding to noise impulses than that without NLM denoising shown in Figure 11(b).

5.2. Application on Outer Race Fault Detection. The recorded vibration signal from a bearing with an outer race defect is shown in Figure 12. From (16), when the motor rotates at the speed of $1797 \mathrm{rpm}$, the calculated bearing characteristic frequency for an OR fault is $107.36 \mathrm{~Hz}$. Figure 12 shows the time domain of the vibration signal. Figure 13 demonstrates the processing results by the MMA and the hybrid NLM denoising and MMA analyses. As shown in Figures 13(a) and 13(d), both MMA and hybrid NLM denoising and MMA have good capability in impulsive extraction. The corresponding frequency spectrum is also given in Figures 13(b) and 13(e) and the characteristic frequency of the outer race fault $\left(f_{o}=\right.$ $106.9 \mathrm{~Hz})$ and its harmonics $\left(2 \times f_{o}\right.$ and $\left.3 \times f_{o}\right)$ are very obvious. The results insist that the outer race fault is present. However, comparison of Figure 13(d) with Figure 13(a) and Figure 13(e) with Figure 13(b) shows that the hybrid NLM denoising and MMA analysis generates less noise impulses than that with only MMA analysis.

\section{Conclusion}

In this paper, a MMA and a hybrid NLM and MMA are suggested to effectively extract the impulse fault characteristics of vibration signals of roller bearings. First, the MMA, which is designed based on DIF and NOTDIF filters, is introduced as an efficient method to extract the impulses. The lengths of SEs are automatically adapted based on the kurtosis to maximize the number of extracted impulse characteristics. However, in the presence of heavy measuring device noise, using only morphology analysis is difficult to extract the fault impulse characteristics which are laid in the noise impulses. To tackle this problem, a hybrid NLM denoising and MMA is then proposed. The effectiveness of the proposed method is verified through the simulated vibration signal and real rolling bearing fault data. The results verified that, compared to the existing morphological operators such as AVR filter or DIF filter, the proposed MMA has a better performance in extracting fault bearing impulses. 


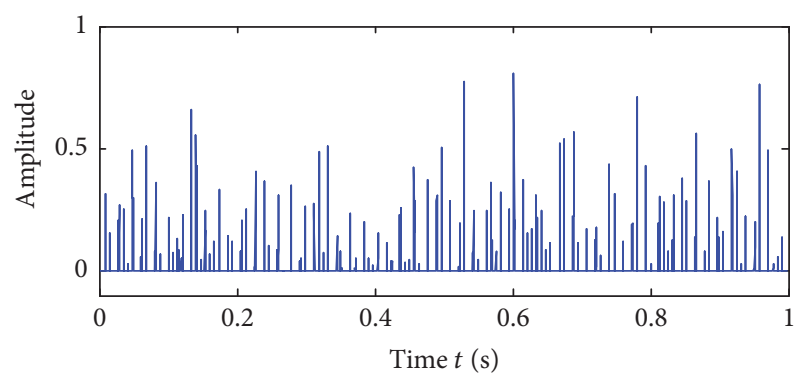

(a)

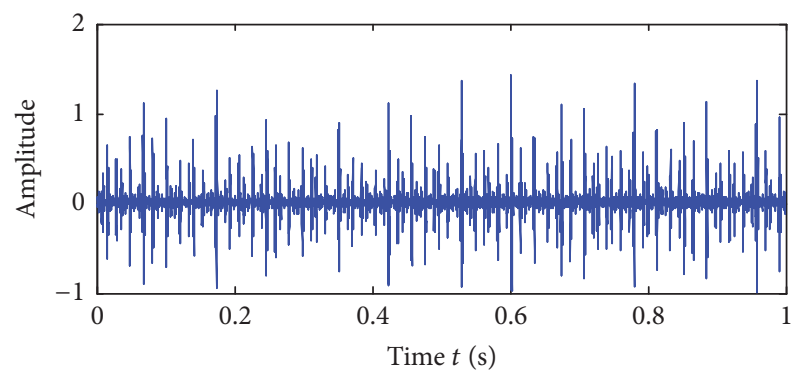

(c)

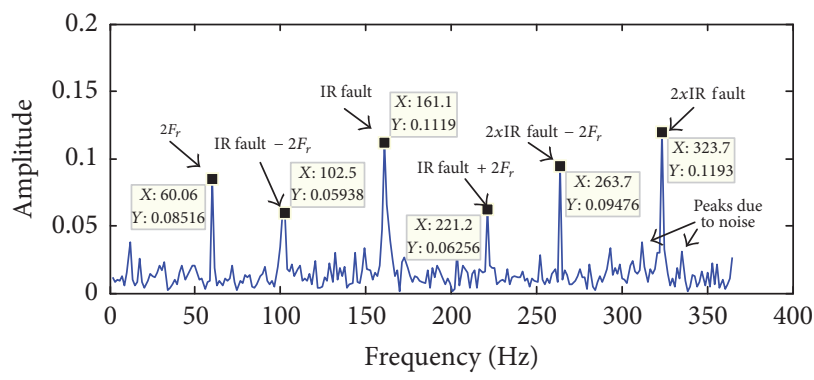

(b)

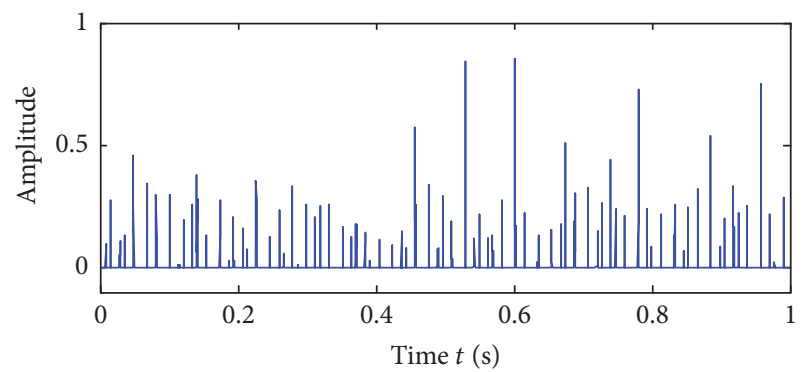

(d)

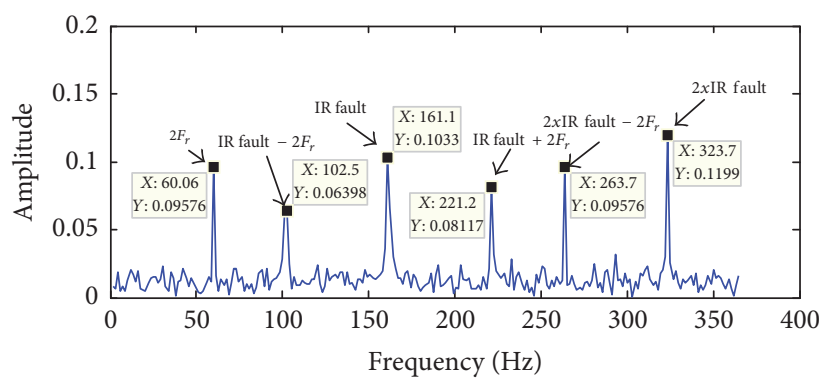

(e)

FIGURE 11: Impulsive extracting results of inner race defect signal in Figure 9: (a) waveform and (b) FFT spectrum of the IR fault signal after Modified-DIF morphology analysis; (c) IR fault signal after NLM denoising; (d) waveform; and (e) FFT spectrum of the IR fault signal after Modified-DIF morphology-based NLM denoising analysis.

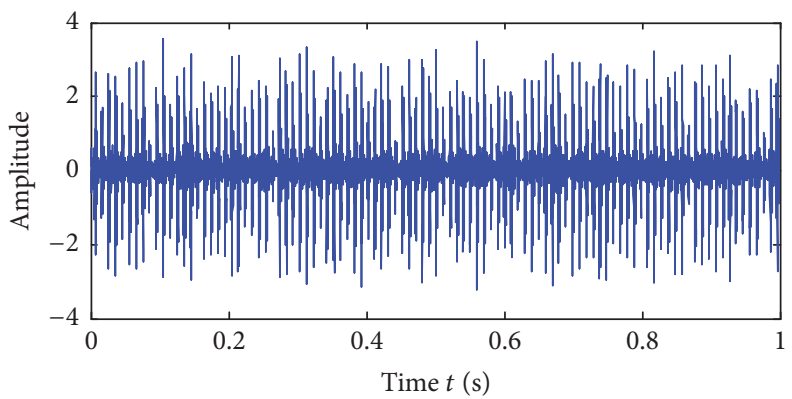

FIGURE 12: Waveform in the time domain of the vibration signal from outer race defect.

The impulses in vibration signal allow diagnosing localized faults effectively; however, they cannot diagnose distributed categories of defects. The vibration signal processing based on pattern recognition has been proved to be effective to diagnose the distributed categories of defects $[10,11]$. Hence, the combining of impulse extraction and patter recognition approaches would be an effective way to enhance the detectability of the system, and this will be a focus of our future work.

\section{Competing Interests}

The authors declare no conflict of interests regarding the publication of this paper. 


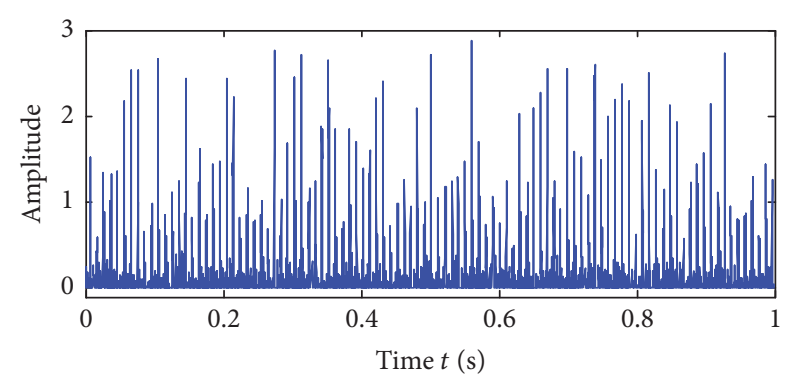

(a)

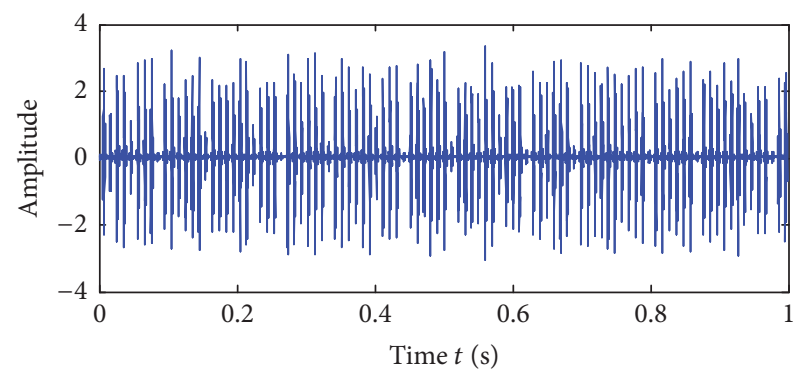

(c)

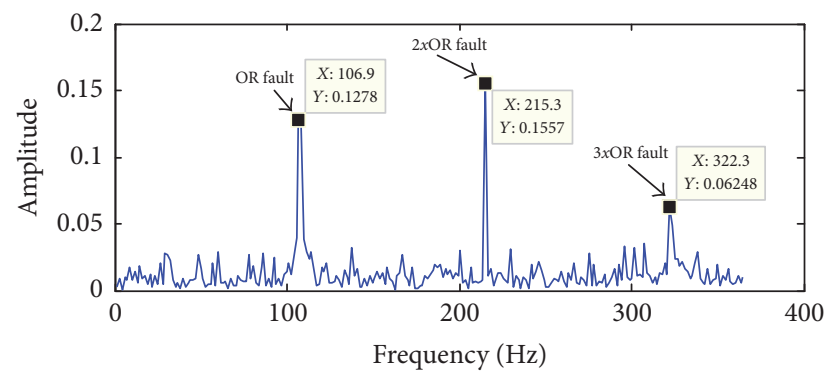

(b)

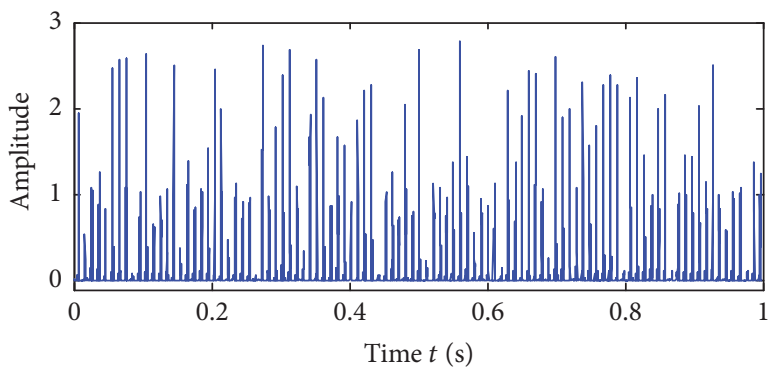

(d)

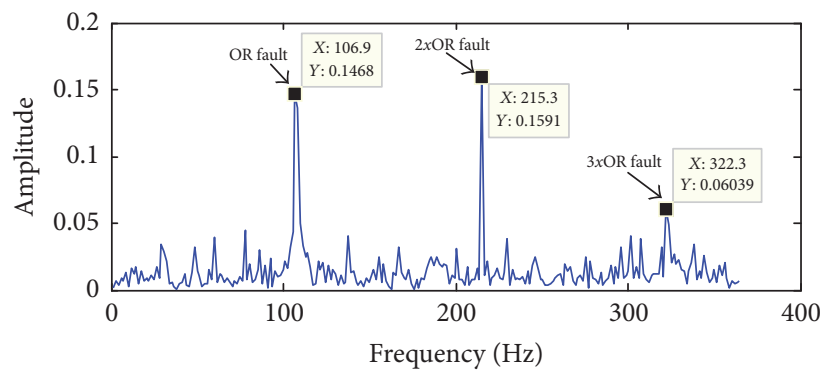

(e)

FIGURE 13: Impulsive extracting results of outer race defect signal in Figure 11: (a) waveform; (b) FFT spectrum of the OR fault signal after Modified-DIF morphology analysis; (c) OR fault signal after NLM denoising; (d) waveform; and (e) FFT spectrum of the OR fault signal after Modified-DIF morphology-based NLM denoising analysis.

\section{Acknowledgments}

This study was partially supported by the UK EPSRS Project EP/K019368/1: Self-Resilient Reconfigurable Assembly Systems with In-Process Quality Improvement.

\section{References}

[1] N. Tandon and B. C. Nakra, "Vibration and acoustic monitoring techniques for the detection of defects in rolling element bearings-a review," Shock \& Vibration Digest, vol. 24, no. 3, pp. 3-11, 1992.

[2] N. Tandon and A. Choudhury, "Review of vibration and acoustic measurement methods for the detection of defects in rolling element bearings," Tribology International, vol. 32, no. 8 , pp. 469-480, 1999.

[3] M. Van, D. Wu, S. S. Ge, and H. Ren, "Fault diagnosis in imagebased visual servoing with eye-in-hand configuration using Kalman filter," IEEE Transactions on Industrial Informatics, 2016.
[4] M. Van, S. S. Ge, and H. Ren, "Robust fault-tolerant control for a class of second-order nonlinear systems using an adaptive third-order sliding mode control," IEEE Transactions on Systems, Man, and Cybernetics: Systems, 2016.

[5] M. Van, S. S. Ge, and H. Ren, "Finite time fault tolerant control for robot manipulators using time delay estimation and continuous nonsingular fast terminal sliding mode control," IEEE Transactions on Cybernetics, 2016.

[6] M. Van, H.-J. Kang, Y.-S. Suh, and K.-S. Shin, "A robust fault diagnosis and accommodation scheme for robot manipulators," International Journal of Control, Automation and Systems, vol. 11, no. 2, pp. 377-388, 2013.

[7] M. Van, H.-J. Kang, and Y.-S. Suh, "A novel neural secondorder sliding mode observer for robust fault diagnosis in robot manipulators," International Journal of Precision Engineering and Manufacturing, vol. 14, no. 3, pp. 397-406, 2013.

[8] M. Van, P. Franciosa, and D. Ceglarek, "Fault diagnosis and fault-tolerant control of uncertain robot manipulators using high-order sliding mode," Mathematical Problems in Engineering, vol. 2016, Article ID 7926280, 14 pages, 2016. 
[9] J. Seshadrinath, B. Singh, and B. K. Panigrahi, "Investigation of vibration signatures for multiple fault diagnosis in variable frequency drives using complex wavelets," IEEE Transactions on Power Electronics, vol. 29, no. 2, pp. 936-945, 2014.

[10] M. Van and H.-J. Kang, "Wavelet kernel local fisher discriminant analysis with particle swarm optimization algorithm for bearing defect classification," IEEE Transactions on Instrumentation and Measurement, vol. 64, no. 12, pp. 3588-3600, 2015.

[11] M. Van and H.-J. Kang, "Bearing defect classification based on individual wavelet local fisher discriminant analysis with particle swarm optimization," IEEE Transactions on Industrial Informatics, vol. 12, no. 1, pp. 124-135, 2016.

[12] D. He, R. Li, and J. Zhu, "Plastic bearing fault diagnosis based on a two-step data mining approach," IEEE Transactions on Industrial Electronics, vol. 60, no. 8, pp. 3429-3440, 2013.

[13] Y. Lei, J. Lin, Z. He, and Y. Zi, "Application of an improved kurtogram method for fault diagnosis of rolling element bearings," Mechanical Systems and Signal Processing, vol. 25, no. 5, pp. 1738-1749, 2011.

[14] V. C. M. N. Leite, J. G. Borges Da Silva, G. F. C. Veloso et al., "Detection of localized bearing faults in induction machines by spectral kurtosis and envelope analysis of stator current," IEEE Transactions on Industrial Electronics, vol. 62, no. 3, pp. 18551865, 2015.

[15] J. Chen, Z. Li, J. Pan et al., "Wavelet transform based on inner product in fault diagnosis of rotating machinery: a review," Mechanical Systems and Signal Processing, vol. 70-70, pp. 1-35, 2016.

[16] M. Van and H.-J. Kang, "Two-stage feature selection for bearing fault diagnosis based on dual-tree complex wavelet transform and empirical mode decomposition," Proceedings of the Institution of Mechanical Engineers, Part C: Journal of Mechanical Engineering Science, vol. 230, no. 2, pp. 291-302, 2016.

[17] W. Su, F. Wang, H. Zhu, Z. Zhang, and Z. Guo, "Rolling element bearing faults diagnosis based on optimal Morlet wavelet filter and autocorrelation enhancement," Mechanical Systems and Signal Processing, vol. 24, no. 5, pp. 1458-1472, 2010.

[18] M. Van, H.-J. Kang, and K.-S. hin, "Rolling element bearing fault diagnosis based on non-local means de-noising and empirical mode decomposition," IET Science, Measurement and Technology, vol. 8, no. 6, pp. 571-578, 2014.

[19] A. Y. Goharrizi and N. Sepehri, "Internal leakage detection in hydraulic actuators using empirical mode decomposition and hilbert spectrum," IEEE Transactions on Instrumentation and Measurement, vol. 61, no. 2, pp. 368-378, 2012.

[20] A. Soualhi, K. Medjaher, and N. Zerhouni, "Bearing health monitoring based on hilbert-huang transform, support vector machine, and regression," IEEE Transactions on Instrumentation and Measurement, vol. 64, no. 1, pp. 52-62, 2015.

[21] M. H. Sedaaghi, "ECG wave detection using morphological filters," Applied Signal Processing, vol. 5, no. 3, pp. 182-194, 1998.

[22] Y. Sun, K. L. Chan, and S. M. Krishnan, "ECG signal conditioning by morphological filtering," Computers in Biology and Medicine, vol. 32, no. 6, pp. 465-479, 2002.

[23] J. F. Zhang, J. S. Smith, and Q. H. Wu, "Morphological undecimated wavelet decomposition for fault location on power transmission lines," IEEE Transactions on Circuits and Systems I: Regular Papers, vol. 53, no. 6, pp. 1395-1402, 2006.

[24] N. G. Nikolaou and I. A. Antoniadis, "Application of morphological operators as envelope extractors for impulsive-type periodic signals," Mechanical Systems and Signal Processing, vol. 17, no. 6, pp. 1147-1162, 2003.
[25] L. Zhang, J. Xu, J. Yang, D. Yang, and D. Wang, "Multiscale morphology analysis and its application to fault diagnosis," Mechanical Systems and Signal Processing, vol. 22, no. 3, pp. 597610, 2008.

[26] J. Wang, G. Xu, Q. Zhang, and L. Liang, "Application of improved morphological filter to the extraction of impulsive attenuation signals," Mechanical Systems and Signal Processing, vol. 23, no. 1, pp. 236-245, 2009.

[27] B. Li, P.-L. Zhang, Z.-J. Wang, S.-S. Mi, and D.-S. Liu, "A weighted multi-scale morphological gradient filter for rolling element bearing fault detection," ISA Transactions, vol. 50, no. 4, pp. 599-608, 2011.

[28] Y. Dong, M. Liao, X. Zhang, and F. Wang, "Faults diagnosis of rolling element bearings based on modified morphological method," Mechanical Systems and Signal Processing, vol. 25, no. 4, pp. 1276-1286, 2011.

[29] A. S. Raj and N. Murali, "Early classification of bearing faults using morphological operators and fuzzy inference," IEEE Transactions on Industrial Electronics, vol. 60, no. 2, pp. 567-574, 2013.

[30] A. Buades, B. Coll, and J. M. Morel, "A review of image denoising algorithms, with a new one," Multiscale Modeling \& Simulation, vol. 4, no. 2, pp. 490-530, 2005.

[31] T. Tasdizen, "Principal neighborhood dictionaries for nonlocal means image denoising," IEEE Transactions on Image Processing, vol. 18, no. 12, pp. 2649-2660, 2009.

[32] D. Van De Ville and M. Kocher, "SURE-based non-local means," IEEE Signal Processing Letters, vol. 16, no. 11, pp. 973-976, 2009.

[33] B. H. Tracey and E. L. Miller, "Nonlocal means denoising of ECG signals," IEEE Transactions on Biomedical Engineering, vol. 59, no. 9, pp. 2383-2386, 2012.

[34] J. Kennedy and R. Eberhart, "Particle swarm optimization," in Proceedings of the IEEE International Conference on Neural Networks, pp. 1942-1948, Perth, Australia, December 1995.

[35] M. Van and H.-J. Kang, "Bearing-fault diagnosis using nonlocal means algorithm and empirical mode decompositionbased feature extraction and two-stage feature selection," IET Science, Measurement and Technology, vol. 9, no. 6, pp. 671-680, 2015.

[36] Z. Liu, H. Cao, X. Chen, Z. He, and Z. Shen, "Multi-fault classification based on wavelet SVM with PSO algorithm to analyze vibration signals from rolling element bearings," Neurocomputing, vol. 99, no. 1, pp. 399-410, 2013.

[37] J. Serra, Image Analysis and Mathematical Morphology, Academic Press, New York, NY, USA, 1982.

[38] P. Maragos and R. W. Schafer, "Morphological filters-part I: their set-theoretic analysis and relations to linear shiftinvariant filters," IEEE Transactions on Acoustics, Speech and Signal Processing, vol. 35, no. 8, pp. 1153-1169, 1987.

[39] K. A. Loparo, Bearing Data Center, Case Western Reserve University, Cleveland, Ohio, USA. 


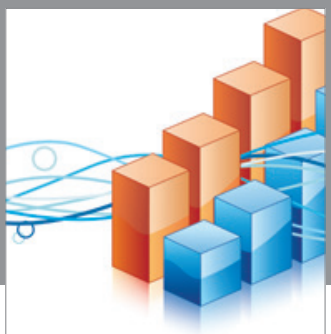

Advances in

Operations Research

vatem alat4

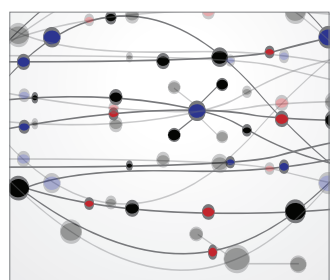

\section{The Scientific} World Journal
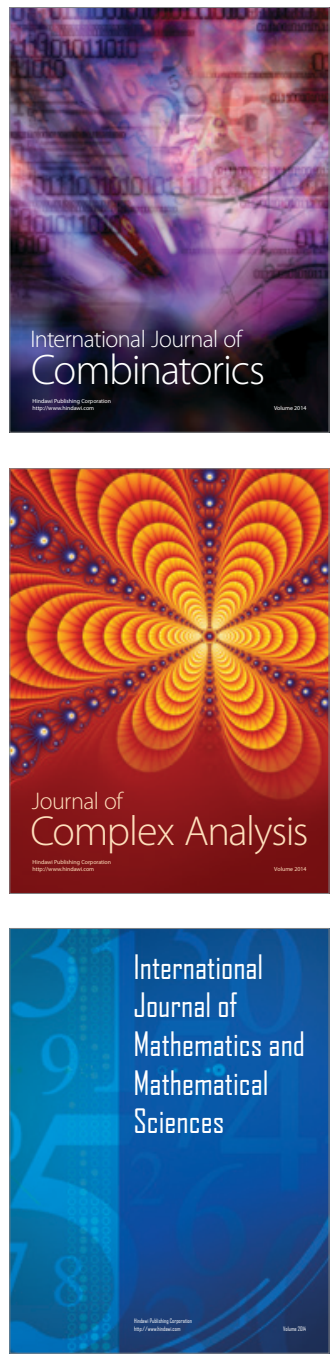
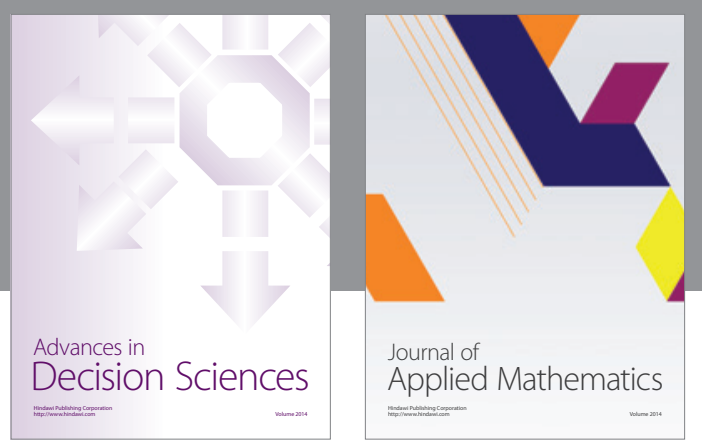

Algebra

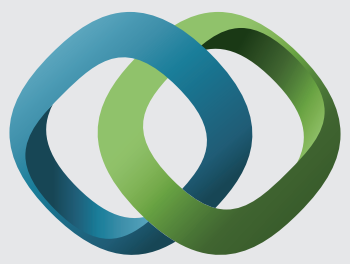

\section{Hindawi}

Submit your manuscripts at

http://www.hindawi.com
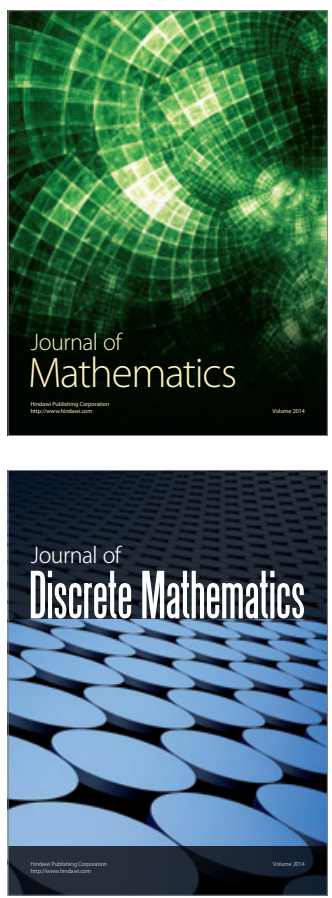

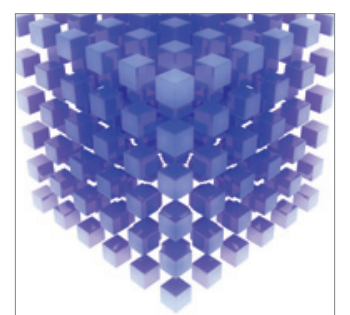

Mathematical Problems in Engineering
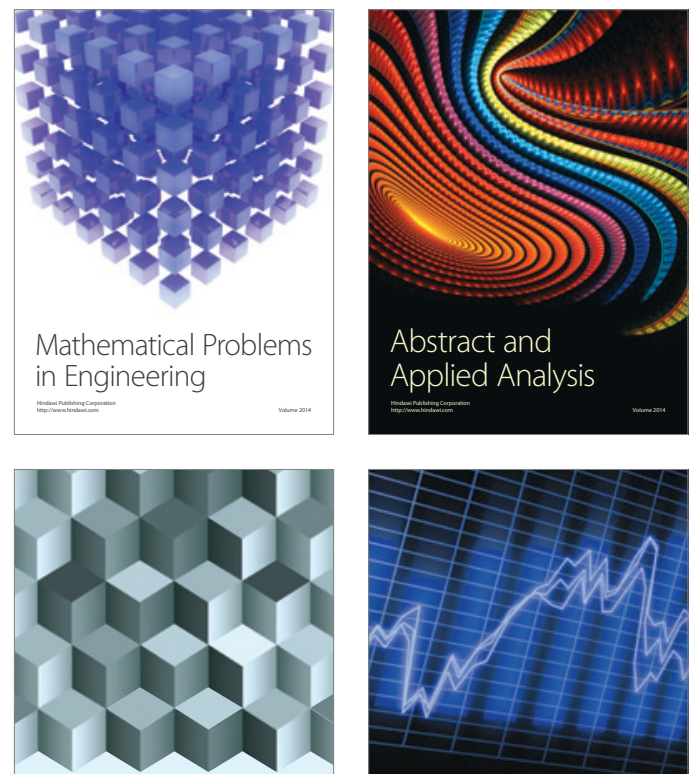

Journal of

Function Spaces

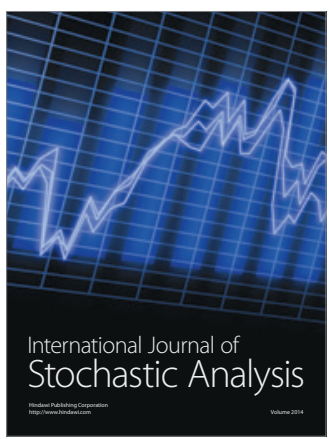

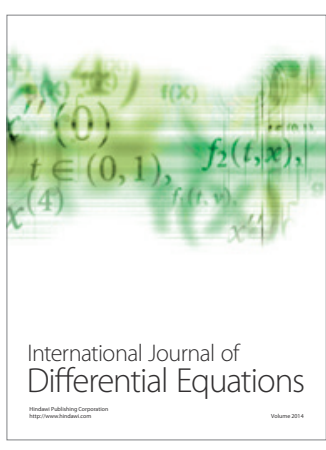
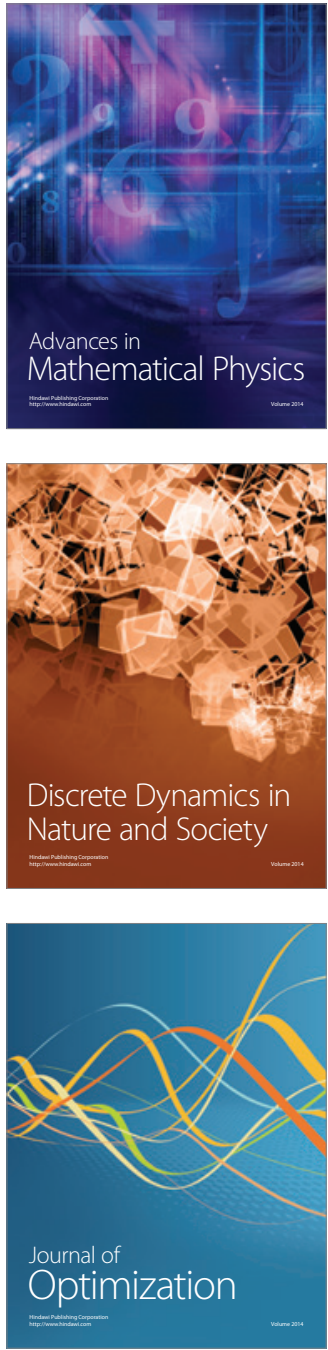